\title{
Asymptotic behavior of impulsive non- autonomous delay difference equations with continuous variables
}

\author{
Danhua $\mathrm{He}^{1^{*}}$ and Qing $\mathrm{Ma}^{2}$
}

\author{
* Correspondence: danhuahe@zisu. \\ edu.cn \\ ${ }^{1}$ Department of Mathematics, \\ Zhejiang International Studies \\ University, Hangzhou, 310012, P. R. \\ China \\ Full list of author information is \\ available at the end of the article
}

\begin{abstract}
In this article, a class of impulsive non-autonomous delay difference equations with continuous variables is considered. By establishing a delay difference inequality with impulsive initial conditions and using the decomposition approach, we obtain the attracting and invariant sets of the equations.
\end{abstract}

Keywords: attracting set, invariant set, non-autonomous, difference inequality, impulsive

\section{Introduction}

Recently, there has been an increasing interest in the study of the asymptotic behavior and other behaviors of the difference equations with continuous variables in which the unknown function is a function of a continuous variable. In particular, delay effects on the asymptotic behavior and other behaviors of this kind of equations have widely been studied in the literature [1-7].

However, besides delay effects, impulsive effects likewise exist in a wide variety of evolutionary process, in which states are changed abruptly at certain moments of time. Recently, impulsive delay difference equations have extensively been studied by many authors. For example, the reader is referred to [8-13]. Unfortunately, impulsive delay difference equations with continuous variables are not well developed due to some theoretical and technical difficulties. Some sufficient conditions for the existence of the invariant and attracting sets of impulsive delay difference equations with continuous variables are obtained in $[14,15]$. To the best of the authors' knowledge, there are no results on the corresponding problems for impulsive non-autonomous delay difference equations with continuous variables. With motivation from the above discussions, this article is devoted to the discussion of this problem. By establishing a delay difference inequality with impulsive initial conditions and using the decomposition approach, we obtain the attracting and invariant sets of the equations.

\section{Preliminaries}

Let $\mathbb{R}^{n}\left(\mathbb{R}_{+}^{n}\right)$ be the space of $n$-dimensional (non-negative) real column vectors, $\mathbb{R}^{m \times n}\left(\mathbb{R}_{+}^{m \times n}\right)$ be the set of $m \times n$ (non-negative) real matrices, $E$ be the $n$-dimensional unit matrix, and $|\cdot|$ be the Euclidean norm of $\mathbb{R}^{n}$. For $A, B \in \mathbb{R}^{m \times n}$ or $A, B \in \mathbb{R}^{n}$,

(c) $2012 \mathrm{He}$ and Ma; licensee Springer. This is an Open Access article distributed under the terms of the Creative Commons Attribution License (http://creativecommons.org/licenses/by/2.0), which permits unrestricted use, distribution, and reproduction in any medium, provided the original work is properly cited. 
$A \geq B(A \leq B, A>B, A<B)$ means that each pair of corresponding elements of $A$ and $B$ satisfies the inequality " $\geq(\leq,>,<)$ ". Especially, $A$ is called a non-negative matrix if $A \geq 0$, and $z$ is called a positive vector if $z>0 . \mathcal{N} \triangleq\{1,2, \ldots n\}$ and $\varrho(A)$ denotes the spectral radius of $A$.

$C[X, Y]$ denotes the space of continuous mappings from the topological space $X$ to the topological space $Y$. Especially, let $C \triangleq C\left[\left[-\tau_{2}, 0\right], \mathbb{R}^{n}\right]$, where $\tau_{2}>0$.

$$
P C\left[\mathbb{J}, \mathbb{R}^{n}\right]=\left\{\begin{array}{l|l}
\psi: \mathbb{J} \rightarrow \mathbb{R}^{n} & \begin{array}{l}
\psi(s) \text { is continuous for all but at most countable points } s \in \mathbb{J} \\
\text { and at these points } s \in \mathbb{J}, \psi\left(s^{+}\right) \text {and } \psi\left(s^{-}\right) \text {exist, } \psi(s)=\psi\left(s^{+}\right)
\end{array}
\end{array}\right\},
$$

where $\mathbb{I} \subset \mathbb{R}$ is an interval, and $\psi\left(s^{+}\right)$and $\psi\left(s^{-}\right)$denote the right- and left-hand limits of the the function $\psi(s)$, respectively. Especially, let $P C \triangleq P C\left[\left[-\tau_{2}, 0\right], \mathbb{R}^{n}\right]$.

In this article, we consider the following impulsive non-autonomous delay difference equation with continuous variable

$$
\left\{\begin{array}{l}
x_{i}(t)=a_{i}(t) x_{i}\left(t-\tau_{1}\right)+\sum_{j=1}^{n} a_{i j}(t) f_{j}\left(\left(x_{j}\left(t-\tau_{2}\right)\right), \quad t \neq t_{k}, t \geq t_{0}, \quad i \in \mathcal{N},\right. \\
x_{i}(t)=J_{i k}\left(x_{i}\left(t^{-}\right)\right), \quad t \geq t_{0}, t=t_{k}, k=1,2, \ldots, \quad i \in \mathcal{N}, \\
x_{i}\left(t_{0}+\theta\right)=\phi_{i}(\theta), \quad \phi_{i} \in P C\left[\left[-\tau_{2}, 0\right], \mathbb{R}\right], \theta \in\left[-\tau_{2}, 0\right]
\end{array}\right.
$$

where $a_{i}(t)=a_{i} h(t)$ and $a_{i j}(t)=a_{i j} h(t), a_{i}$ and $a_{i j}$ are real constants, $h(t) \leq 1$ is a positive integral function and satisfies $\sup _{t \geq t_{0}} \int_{t-\tau_{2}}^{t} h(s) d s=H_{2}<\infty$ and $\lim _{t \rightarrow \infty} \int_{t_{0}}^{t} h(s) d s=\infty$. $\tau_{1}$ and $\tau_{2}$ are positive real numbers such that $\tau_{1}<\tau_{2} . t_{k}(k=1,2, \ldots)$ is an impulsive sequence such that $t_{1}<t_{2}<\ldots, \lim _{k \rightarrow \infty} t_{k}=\infty$. $f_{j}$ and $J_{i k}: \mathbb{R} \rightarrow \mathbb{R}$ are real-valued functions. Moreover, we assume that $f_{j}(0)=0$ and $J_{i k}(0)=0$, then system (1) admits an equilibrium solution $x(t) \equiv 0$.

By the solution of (1), we mean a piecewise continuous real-valued function $x_{i}(t)$ defined on the interval $\left[t_{0}-\tau_{2}, \infty\right)$ which satisfies (1) for all $t \geq t_{0}$.

By the method of steps, one can easily see that, for any given initial function $\varphi_{i} \in P C$ $\left[\left[-\tau_{2}, 0\right], \mathbb{R}\right]$, there exists a unique solution $x_{i}(t), i \in \mathcal{N}$, of $(1)$.

For convenience, we rewrite the system (1) as the following vector form

$$
\left\{\begin{array}{l}
x(t)=h(t)\left[A_{0} x\left(t-\tau_{1}\right)+A f\left(x\left(t-\tau_{2}\right)\right)\right], \quad t \neq t_{k}, t \geq t_{0} \\
x(t)=J_{k}\left(x\left(t^{-}\right)\right), \quad t \geq t_{0}, \quad t=t_{k}, k=1,2, \ldots \\
x\left(t_{0}+\theta\right)=\phi(\theta), \quad \theta \in\left[-\tau_{2}, 0\right]
\end{array}\right.
$$

where $x(t)=\left(x_{1}(t), \ldots, x_{n}(t)\right)^{T}, A_{0}=\operatorname{diag}\left\{a_{1}, \ldots, a_{n}\right\}, A=\left(a_{i j}\right)_{n \times n}, f(x)=\left(f_{1}\left(x_{1}\right), \ldots, f_{n}\left(x_{n}\right)\right)^{T}$, $J_{k}(x)=\left(J_{1 k}\left(x_{1}\right), \ldots, J_{n k}\left(x_{n}\right)\right)^{T}$, and $\varphi=\left(\varphi_{1}, \ldots, \varphi_{n}\right)^{T} \in P C$.

Definition 2.1. The set $S \subset P C$ is called a positive invariant set of (2), if for any initial value $\varphi \in S$, the solution $x_{t}\left(t_{0}, \varphi\right) \in S, t \geq t_{0}$, where $x_{t}\left(t_{0}, \varphi\right)=x\left(t+s, t_{0}, \varphi\right) \in$ $P C, s \in\left[-\tau_{2}, 0\right]$.

Definition 2.2. The set $S \subset P C$ is called a global attracting set of (2), if for any initial value $\varphi \in P C$, the solution $x\left(t, t_{0}, \varphi\right)$ satisfies

$$
\operatorname{dist}\left(x\left(t, t_{0}, \phi\right), S\right) \rightarrow 0, \text { as } t \rightarrow+\infty,
$$

where $\operatorname{dist}(\varphi, S)=\inf _{\psi \in S} \operatorname{dist}(\varphi, \psi), \operatorname{dist}(\varphi, \psi)=\sup _{\theta \in\left[-\tau_{2,0]}\right.}|\varphi(\theta)-\psi(\theta)|$, for $\psi \in P C$.

Definition 2.3. System (2) is said to be globally exponentially stable if for any solution $x\left(t, t_{0}, \varphi\right)$, there exist constants $\lambda_{0}>0$ and $\kappa_{0} \geq 0$ such that, 


$$
\left|x\left(t, t_{0}, \phi\right)\right| \leq \kappa_{0}|| \phi|| e^{-\lambda_{0}\left(t-t_{0}\right)}, \quad t \geq t_{0},
$$

where $\|\emptyset\|=\sup _{\theta \in\left[-\tau_{2}, 0\right]}|\emptyset(s)|$.

Following [16], we split the matrices $A_{0}, A$ into two parts, respectively,

$$
A_{0}=A_{0}^{+}-A_{0}^{-}, \quad A=A^{+}-A^{-}
$$

with $a_{i}^{+}=\max \left\{a_{i}, 0\right\}, a_{i}^{-}=\max \left\{-a_{i}, 0\right\}, a_{i j}^{+}=\max \left\{a_{i j}, 0\right\}, a_{i j}^{-}=\max \left\{-a_{i j}, 0\right\}$.

Set $y=-x, g(u)=-f(-u)$. Then, by a similar argument with [15], we can get the following equations from the first equation of (2)

$$
v(t)=h(t)\left[\mathcal{A}_{0} v\left(t-\tau_{1}\right)+\mathcal{A} \xi\left(v\left(t-\tau_{2}\right)\right)\right],
$$

where

$$
v(t)=\left\{\begin{array}{l}
x(t) \\
y(t)
\end{array}\right\}, \quad \xi(v(t))=\left\{\begin{array}{l}
f(x(t)) \\
g(y(t))
\end{array}\right\}, \quad \mathcal{A}_{0}=\left\{\begin{array}{l}
A_{0}^{+} A_{0}^{-} \\
A_{0}^{-} A_{0}^{+}
\end{array}\right\}, \quad \mathcal{A}=\left\{\begin{array}{l}
A^{+} A^{-} \\
A^{-} A^{+}
\end{array}\right\} .
$$

Set $I_{k}(u)=-J_{k}(-u)$; in view of the impulsive part of (2), we have

$$
v\left(t_{k}\right)=\omega_{k}\left(v\left(t_{k}^{-}\right)\right), k=1,2, \ldots,
$$

where $\omega_{k}(v)=\left(J_{k}(x)^{T}, I_{k}\left(y(t)^{T}\right)^{T}\right.$.

Lemma 2.1. $[17,18]$ If $M \in \mathbb{R}_{+}^{n \times n}$ and $\mathrm{\varrho}(M)<1$, then $(E-M)^{-1} \geq 0$.

Lemma 2.2. [18]Suppose that $M \in \mathbb{R}_{+}^{n \times n}$ and $\mathrm{\varrho}(\mathrm{M})<1$, then there exists a positive vector $z$ such that $(E-M) z>0$.

For $M \in \mathbb{R}_{+}^{n \times n}$ and $\varrho(M)<1$, we denote

$$
\Omega_{\varrho}(M)=\left\{z \in \mathbb{R}^{n} \mid(E-M) z>0, z>0\right\} .
$$

In order to discuss the asymptotic behavior of (2), and for the brevity of later discussion, we list all our conditions as follows.

$\left(A_{1}\right)$ For any $x, y \in \mathbb{R}^{n}$, there exists a non-negative diagonal matrix $\bar{P}$ and vector $\mu=\left(\mu_{1}, \ldots, \mu_{n}\right)^{T} \geq 0$ such that

$$
f(x)-f(y) \leq \bar{P}(x-y)+\mu .
$$

$\left(A_{2}\right)$ For any $x, y \in \mathbb{R}^{n}$, there exist nonnegative matrices $R_{k}$ such that

$$
J_{k}(x)-J_{k}(y) \leq R_{k}(x-y), \quad k=1,2, \ldots
$$

$\left(A_{3}\right)$ Let $\varrho\left(\mathcal{A}_{0}+\mathcal{A P}\right)<1$, where $\mathcal{P}=\operatorname{diag}\{\bar{P}, \bar{P}\}$.

$\left(A_{4}\right)$ There exists a constant $\gamma$ such that

$$
\frac{\ln \gamma_{k}}{\int_{t_{k-1}}^{t_{k}} h(s) d s} \leq \gamma<\lambda, \quad k=1,2, \ldots
$$

where the scalar $\lambda$ satisfies $0<\lambda$ and is determined by the following inequality

$$
\left(\mathcal{A}_{0} e^{\lambda H_{1}}+\mathcal{A} \mathcal{P} e^{\lambda H_{2}}-E\right) z \leq 0,
$$


where $z=\left(z_{1}, \ldots, z_{2 n}\right)^{T} \in \Omega_{\varrho}\left(\mathcal{A}_{0}+\mathcal{A P}\right)$, and

$$
\gamma_{k} \geq 1 \text { and } \gamma_{k} z \geq \mathcal{R}_{k} z, \mathcal{R}_{k}=\operatorname{diag}\left\{R_{k}, R_{k}\right\}, k=1,2, \ldots
$$

$\left(A_{5}\right)$ Let

$$
\sigma=\sum_{k=1}^{\infty} \ln \sigma_{k}<\infty, \quad k=1,2, \ldots,
$$

where $\sigma_{k} \geq 1$ satisfy

$$
\mathcal{R}_{k}\left(E-\mathcal{A}_{0}-\mathcal{A P}\right)^{-1} \mathcal{A} \Lambda \leq \sigma_{k}\left(E-\mathcal{A}_{0}-\mathcal{A P}\right)^{-1} \mathcal{A} \Lambda,
$$

where $\Lambda=\left(\mu^{T}, \mu^{T}\right)^{T}$.

\section{Main results}

In this section, we will give the main results of this article. The proofs of these results are placed in the following section for the sake of brevity.

Theorem 3.1. Let $P=\left(p_{i j}\right)_{n \times n}, W=\left(w_{i j}\right)_{n \times n} \in \mathbb{R}_{+}^{n \times n}, I=\left(I_{1}, \ldots, I_{n}\right)^{T} \in \mathbb{R}_{+}^{n}$, and $u(t)$ $\in \mathbb{R}^{n}$ be a solution of the following delay difference inequality with the initial condition $u\left(t_{0}+\theta\right)=\varphi(\theta), \theta \in\left[-\tau_{2}, 0\right] \varphi \in P C$,

$$
u(t) \leq h(t)\left[P u\left(t-\tau_{1}\right)+W u\left(t-\tau_{2}\right)+I\right], \quad t \geq t_{0},
$$

where $\tau_{2}>\tau_{1}, h(t) \leq 1$ is a positive integral function and $\lim _{t \rightarrow \infty} \int_{t_{0}}^{t} h(s) d s=\infty$ and $\lim _{t \rightarrow \infty} \int_{t_{0}}^{t} h(s) d s=\infty$.

If $\mathrm{\varrho}(P+W)<1$, then there exists a positive vector $z=\left(z_{1}, z_{2}, \ldots, z_{n}\right)^{T}$ such that

$$
u(t) \leq \kappa z e^{-\lambda \int_{t_{0}}^{t} h(s) d s}+(E-P-W)^{-1} I, \quad t \geq t_{0},
$$

provided that the initial condition satisfies

$$
u(t) \leq \kappa z e^{-\lambda \int_{t_{0}}^{t} h(s) d s}+(E-P-W)^{-1} I, \quad \kappa \geq 0, t \in\left[t_{0}-\tau_{2}, t_{0}\right],
$$

where the positive number $\lambda>0$ is determined by the following inequality

$$
\left(P e^{\lambda H_{1}}+W e^{\lambda H_{2}}-E\right) z \leq 0,
$$

where $H_{1} \sup _{t \geq t_{0}} \int_{t-\tau_{1}}^{t} h(s) d s$. Clearly, $H_{1}<H_{2}<\infty$.

Theorem 3.2. If $\left(A_{1}\right)-\left(A_{5}\right)$ hold, then $S=\left\{\phi \in P C \mid-e^{\sigma} \mathcal{N}_{2} \leq \phi \leq e^{\sigma} \mathcal{N}_{1}\right\}$ is a global attracting set of (2), where $\mathcal{N}_{1}, \mathcal{N}_{2} \in \mathbb{R}^{n}$ and $\left(\mathcal{N}_{1}^{T}, \mathcal{N}_{2}^{T}\right)^{T}=\hat{N}=\left(E-\mathcal{A}_{0}-\mathcal{A P}\right)^{-1} \mathcal{A} \Lambda$.

Theorem 3.3. If $\left(A_{1}\right)-\left(A_{3}\right)$ with $R_{k} \leq E$ hold, then $S=\left\{\phi \in P C \mid-\mathcal{N}_{2} \leq \phi \leq \mathcal{N}_{1}\right\}$ is a positive invariant set and also a global attracting set of (2), where $\mathcal{N}_{1}, \mathcal{N}_{2} \in \mathbb{R}^{n}$ and $\left(\mathcal{N}_{1}^{T}, \mathcal{N}_{2}^{T}\right)^{T}=\hat{N}=\left(E-\mathcal{A}_{0}-\mathcal{A P}\right)^{-1} \mathcal{A} \Lambda$

For the case $I=0$, we easily observe $x(t) \equiv 0$ is a solution of (2) from $\left(A_{1}\right)$ and $\left(A_{2}\right)$. In the following, we give the attractivity of the zero solution and the proof is similar to that of Theorem 3.2.

Corollary 3.1. If $\left(A_{1}\right)-\left(A_{4}\right)$ hold with $I=0$, then the zero solution of (2) is globally exponentially stable. 


\section{Proofs of main results}

Proof of Theorem 3.1. Since $P, W \in \mathbb{R}_{+}^{n \times n}$ and $\varrho(P+W)<1$, by Lemma 2.2, there exists a positive vector $z \in \Omega_{\varrho}(P+W)$ such that $\left.(E-P-W)\right) z>0$. By continuity, we know that (15) has at least one positive solution $\lambda$, i.e.,

$$
\sum_{j=1}^{n}\left[p_{i j} e^{\lambda H_{1}}+w_{i j} e^{\lambda H_{2}}\right] z_{j} \leq z_{i}, \quad i \in \mathcal{N} .
$$

Set

$$
u(t)=v(t) e^{-\lambda \int_{t_{0}}^{t} h(s) d s}+N, \quad t \geq t_{0}-\tau_{2},
$$

where $N=(E-P-W)^{-1} I$; substituting (17) into (12), we have

$$
v(t) e^{-\lambda \int_{t_{0}}^{t} h(s) d s}+N \leq h(t)\left[P\left(v\left(t-\tau_{1}\right) e^{-\lambda \int_{t_{0}}^{t-\tau_{1}} h(s) d s}+N\right)+W\left(v\left(t-\tau_{2}\right) e^{-\lambda \int_{t_{0}}^{t-\tau_{2}} h(s) d s}+N\right)+I\right] .
$$

Then, by (18) and $h(t) \leq 1$, we obtain

$$
v(t) \leq h(t)\left[P v\left(t-\tau_{1}\right) e^{\lambda \int_{t-\tau_{1}}^{t} h(s) d s}+W v\left(t-\tau_{2}\right) e^{\lambda \int_{t-\tau_{2}}^{t} h(s) d s}\right] .
$$

By (14) and (17), we get that

$$
v(\theta) \leq \kappa z, \theta \in\left[t_{0}-\tau_{2}, t_{0}\right]
$$

Next, we will prove for any $t \geq t_{0}$,

$$
v(t) \leq \kappa z .
$$

To prove (21), we first prove that for any positive constant $\varepsilon$,

$$
v(t)<(1+\varepsilon) \kappa z, t \geq t_{0} .
$$

If (22) is not true, then there must be a $t^{*}>t_{0}$ and some integer $r$ such that

$$
v(t)<(1+\varepsilon) \kappa z, \text { for } t \in\left[t_{0}, t^{*}\right), \quad v_{r}\left(t^{*}\right)=(1+\varepsilon) \kappa z_{r} .
$$

By using (16) and (19), we obtain that

$$
\begin{aligned}
(1+\varepsilon) \kappa z_{r}=v_{r}\left(t^{*}\right) & \leq h\left(t^{*}\right) \sum_{j=1}^{n}\left[p_{r j} v_{j}\left(t^{*}-\tau_{1}\right) e^{\lambda \int_{t^{*}-\tau_{1}}^{t^{*}} h(s) d s}+w_{r j} v_{j}\left(t^{*}-\tau_{2}\right) e^{\lambda \int_{t^{*}-\tau_{2}}^{t^{*}} h(s) d s}\right] \\
& <h\left(t^{*}\right) \sum_{j=1}^{n}\left[p_{r j} e^{\lambda \int_{t^{*}-\tau_{1}}^{\int^{*}} h(s) d s}+w_{r j} e^{\lambda \int_{t^{*}-\tau_{2}}^{*^{*}} h(s) d s}\right](1+\varepsilon) \kappa z_{j} \\
& \leq h\left(t^{*}\right) \sum_{j=1}^{n}\left[p_{r j} e^{\lambda H_{1}}+w_{r j} e^{\lambda H_{2}}\right](1+\varepsilon) \kappa z_{j} \\
& \leq(1+\varepsilon) \kappa z_{r}
\end{aligned}
$$

which is a contradiction. Hence, (22) holds for all numbers $\varepsilon>0$; it follows immediately that (21) is always satisfied, which can easily be led to (13). This completes the proof.

Proof of Theorem 3.2. Since $\varrho\left(\mathcal{A}_{0}+\mathcal{A P}\right)<1$, by Lemma 2.2, there exists a positive vector $z \in \Omega_{\varrho}\left(\mathcal{A}_{0}+\mathcal{A P}\right)$ such that $\left(E-\left(\mathcal{A}_{0}+\mathcal{A P}\right)\right) z>0$. Using continuity, we obtain that inequality (8) has at least one positive solution $\lambda$. 
From (5) and Condition (6), we can claim that for any $v \in \mathbb{R}^{2 n}$,

$$
\xi(v) \leq \mathcal{P} v+\Lambda
$$

and

$$
\omega_{k}(v) \leq \mathcal{R}_{k} v\left(t_{k}^{-}\right), k=1,2, \ldots
$$

where $\Lambda=\left(\mu^{T}, \mu^{T}\right)^{T}$.

So by using (3) and (4) and taking into account (24) and (25), we get

$$
v(t) \leq h(t)\left[\mathcal{A}_{0} v\left(t-\tau_{1}\right)+\mathcal{A P} v\left(t-\tau_{2}\right)+\mathcal{A} \Lambda\right]
$$

and

$$
v\left(t_{k}\right) \leq \mathcal{R}_{k} v\left(t_{k}^{-}\right), k=1,2, \ldots
$$

respectively.

Noting $\varrho\left(\mathcal{A}_{0}+\mathcal{A P}\right)<1$ and $\mathcal{A}_{0}, \mathcal{A P} \in \mathbb{R}_{+}^{n \times n}$, then, by Lemma 2.1 , we can get $\left(E-\mathcal{A}_{0}-\mathcal{A P}\right)^{-1} \geq 0$ and $\hat{N} \triangleq\left(E-\mathcal{A}_{0}-\mathcal{A P}\right)^{-1} \mathcal{A} \Lambda \geq 0$.

For the initial conditions: $x\left(t_{0}+\theta\right)=\varphi(\theta), \theta \in\left[-\tau_{2}, 0\right]$, where $\varphi \in P C$, we have

$$
v(t) \leq \kappa_{0} z e^{-\lambda \int_{t_{0}}^{t} h(s) d s} \leq \kappa_{0} z e^{-\lambda \int_{t_{0}}^{t} h(s) d s}+\hat{N}, \quad t \in\left[t_{0}-\tau_{2}, t_{0}\right]
$$

where

$$
\kappa_{0}=\frac{\|\phi\|}{\min _{1 \leq i \leq 2 n}\left\{z_{i}\right\}}, \quad z \in \Omega_{\varrho}\left(\mathcal{A}_{0}+\mathcal{A P}\right) .
$$

Then, all the conditions of Theorem 3.1 are satisfied by (26), (28), and Condition $\left(A_{3}\right)$, we derive that

$$
v(t) \leq \kappa_{0} z e^{-\lambda \int_{t_{0}}^{t} h(s) d s}+\hat{N}, \quad t \in\left[t_{0}, t_{1}\right) .
$$

Suppose for all $\iota=1, \ldots, k$, the inequalities

$$
v(t) \leq \gamma_{0} \cdots \gamma_{\iota-1} \kappa_{0} z e^{-\lambda \int_{t_{0}}^{t} h(s) d s}+\sigma_{0} \cdots \sigma_{l-1} \hat{N}, \quad t \in\left[t_{l-1}, t_{l}\right),
$$

hold, where $\gamma_{0}=\sigma_{0}=1$. Then, from (9), (11), (30), and $\left(A_{2}\right)$, the impulsive part of (2) satisfies that

$$
\begin{aligned}
v\left(t_{k}\right) & \leq \mathcal{R}_{k} v\left(t_{k}^{-}\right) \\
& \leq \mathcal{R}_{k}\left[\gamma_{0} \cdots \gamma_{k-1} \kappa_{0} z e^{-\lambda \int_{t_{0}}^{t_{k}} h(s) d s}+\sigma_{0} \cdots \sigma_{k-1} \hat{N}\right] \\
& \leq \gamma_{0} \cdots \gamma_{k-1} \gamma_{k} \kappa_{0} z e^{-\lambda \int_{t_{0}}^{t_{k}} h(s) d s}+\sigma_{0} \cdots \sigma_{k-1} \sigma_{k} \hat{N}
\end{aligned}
$$

This, together with (30) and $\gamma_{k}, \sigma_{k} \geq 1$, leads to

$$
v(t) \leq \gamma_{0} \cdots \gamma_{k-1} \gamma_{k} \kappa_{0} z e^{-\lambda \int_{t_{0}}^{t} h(s) d s}+\sigma_{0} \cdots \sigma_{k-1} \sigma_{k} \hat{N}, \quad t \in\left[t_{k}-\tau_{2}, t_{k}\right] .
$$

On the other hand,

$$
v(t) \leq h(t)\left[\mathcal{A}_{0} v\left(t-\tau_{1}\right)+\mathcal{A} \mathcal{P} v\left(t-\tau_{2}\right)+\sigma_{0}, \cdots \sigma_{k} \mathcal{A} \Lambda\right], t \neq t_{k} .
$$


It follows from (32)-(33) and Theorem 3.1 that

$$
v(t) \leq \gamma_{0} \cdots \gamma_{k-1} \gamma_{k} \kappa_{0} z e^{-\lambda \int_{t_{0}}^{t} h(s) d s}+\sigma_{0} \cdots \sigma_{k-1} \sigma_{k} \hat{N}, \quad t \in\left[t_{k}, t_{k+1}\right) .
$$

By the mathematical induction, we can conclude that

$$
v(t) \leq \gamma_{0} \cdots \gamma_{k-1} \kappa_{0} z e^{-\lambda \int_{t_{0}}^{t} h(s) d s}+\sigma_{0} \cdots \sigma_{k-1} \hat{N}, \quad t \in\left[t_{k-1}, t_{k}\right), k=1,2, \ldots
$$

From (7) and (10),

$$
\gamma_{k} \leq e^{\gamma \int_{t_{k-1}}^{t_{k}} h(s) d s}, \quad \sigma_{0} \cdots \sigma_{k-1} \leq e^{\sigma},
$$

we can use (35) to conclude that

$$
\begin{aligned}
v(t) & \leq e^{\gamma \int_{t_{0}}^{t_{1}} h(s) d s} \cdots e^{\gamma \int_{t_{k-2}}^{t_{k-1}} h(s) d s} \kappa_{0} z e^{-\lambda \int_{t_{0}}^{t} h(s) d s}+\sigma_{0} \cdots \sigma_{k-1} \hat{N} \\
& \leq \kappa_{0} z e^{\gamma \int_{t_{0}}^{t} h(s) d s} e^{-\lambda \int_{t_{0}}^{t} h(s) d s}+e^{\sigma} \hat{N} \\
& =\kappa_{0} z e^{-(\lambda-\gamma) \int_{t_{0}}^{t} h(s) d s}+e^{\sigma} \hat{N}, t \in\left[t_{k-1}, t_{k}\right), k=1,2, \ldots
\end{aligned}
$$

This implies that the conclusion of the theorem holds and the proof is complete.

Proof of Theorem 3.3. Similarly, the inequality (8) holds by $\left(A_{3}\right)$. For the initial conditions: $x\left(t_{0}+s\right)=\varphi(s), s \in\left[-\tau_{2}, 0\right]$, where $\varphi \in S$, we have

$$
v(t) \leq\left(E-\mathcal{A}_{0}-\mathcal{A P}\right)^{-1} \mathcal{A} \Lambda, \quad t \in\left[t_{0}-\tau_{2}, t_{0}\right] .
$$

By (36) and Theorem 3.1 with $\kappa=0$, we have

$$
v(t) \leq\left(E-\mathcal{A}_{0}-\mathcal{A} \mathcal{P}\right)^{-1} \mathcal{A} \Lambda, \quad t \in\left[t_{0}, t_{1}\right) .
$$

Then, from (27) and $R_{k} \leq E$,

$$
v\left(t_{1}\right) \leq \mathcal{R}_{1} v\left(t_{1}^{-}\right) \leq E v\left(t_{1}^{-}\right) \leq\left(E-\mathcal{A}_{0}-\mathcal{A P}\right)^{-1} \mathcal{A} \Lambda .
$$

Thus,

$$
v(t) \leq\left(E-\mathcal{A}_{0}-\mathcal{A P}\right)^{-1} \mathcal{A} \Lambda, t \in\left[t_{1}-\tau_{2}, t_{1}\right] .
$$

Using Theorem 3.1 again, we obtain

$$
v(t) \leq\left(E-\mathcal{A}_{0}-\mathcal{A} \mathcal{P}\right)^{-1} \mathcal{A} \Lambda, t \in\left[t_{1}, t_{2}\right) .
$$

By introduction, we have

$$
v(t) \leq\left(E-\mathcal{A}_{0}-\mathcal{A P}\right)^{-1} \mathcal{A} \Lambda, t \in\left[t_{k-1}, t_{k}\right), k=1,2, \ldots
$$

Therefore, $S=\left\{\phi \in P C \mid-\mathcal{N}_{2} \leq \phi \leq \mathcal{N}_{1}\right\}$ is a positive invariant set. Since $\mathscr{R}_{k} \leq E$, a direct calculation shows that $\gamma_{k}=\sigma_{k}=1$ and $\sigma=0$ in Theorem 3.2. It follows from Theorem 3.2 that the set $S$ is also a global attracting set of (2). The proof is complete.

\section{Illustrative example}

The following illustrative example will demonstrate the effectiveness of our results. 
Example 4.1 Consider the following impulsive delay difference equation:

$$
\left\{\begin{array}{c}
x_{1}(t)=\left(1+\cos ^{2} t\right)\left[\frac{1}{4} x_{1}(t-1)+\frac{1}{3} x_{2}(t-2)+2\right] \\
x_{2}(t)=\left(1+\cos ^{2} t\right)\left[-\frac{1}{4} x_{2}(t-1)+\frac{1}{5} x_{1}(t-2)+2\right]
\end{array}, t \neq t_{k},\right.
$$

with

$$
\left\{\begin{array}{l}
x_{1}\left(t_{k}\right)=\alpha_{1 k} x_{1}\left(t_{k}^{-}\right)-\beta_{1 k} x_{2}\left(t_{k}^{-}\right) \\
x_{2}\left(t_{k}\right)=\beta_{2 k} x_{1}\left(t_{k}^{-}\right)+\alpha_{2 k} x_{2}\left(t_{k}^{-}\right)
\end{array}\right.
$$

where $\alpha_{i k}$ and $\beta_{i k}$ are non-negative constants, and the impulsive sequence $t_{k}(k=1$, $2, \ldots)$ satisfy: $t_{1}<t_{2}<\cdots, \lim _{k \rightarrow \infty} t_{k}=\infty$.

The parameters of $\left(A_{1}\right)-\left(A_{3}\right)$ are as follows:

$$
\begin{gathered}
A_{0}=\left(\begin{array}{c}
\frac{1}{4} 0 \\
0-\frac{1}{4}
\end{array}\right), A=\left(\begin{array}{l}
0 \frac{1}{3} \\
\frac{1}{5} 0
\end{array}\right), \bar{P}=\left(\begin{array}{l}
10 \\
01
\end{array}\right), \mu=\left(\begin{array}{l}
2 \\
2
\end{array}\right), R_{k}=\left(\begin{array}{ll}
\alpha_{1 k} & \beta_{1 k} \\
\beta_{2 k} & \alpha_{2 k}
\end{array}\right), \\
\mathcal{A}_{0}=\left(\begin{array}{c}
\frac{1}{4} 000 \\
000 \frac{1}{4} \\
00 \frac{1}{4} 0 \\
0 \frac{1}{4} 00
\end{array}\right), \mathcal{A}=\left(\begin{array}{c}
0 \frac{1}{3} 00 \\
\frac{1}{5} 000 \\
000 \frac{1}{3} \\
00 \frac{1}{5} 0
\end{array}\right), \mathcal{R}_{k}=\left(\begin{array}{cccc}
\alpha_{1 k} & \beta_{1 k} & 0 & 0 \\
\beta_{2 k} & \alpha_{2 k} & 0 & 0 \\
0 & 0 & \alpha_{1 k} & \beta_{1 k} \\
0 & 0 & \beta_{2 k} & \alpha_{2 k}
\end{array}\right),
\end{gathered}
$$

and $\mathcal{P}=\operatorname{diag}\{1,1,1,1\}$.

It is easy to prove that $\varrho\left(\mathcal{A}_{0}+\mathcal{A P}\right)=0.5082<1$ and

$$
\Omega_{\varrho}\left(\mathcal{A}_{0}+\mathcal{A} \mathcal{P}\right)=\left\{\begin{array}{l|l}
\left(z_{1}, z_{2}, z_{3}, z_{4}\right)^{T}>0 & \begin{array}{l}
\frac{3}{4} z_{1}-\frac{1}{3} z_{2}>0 \\
-\frac{1}{5} z_{1}+z_{2}-\frac{1}{4} z_{4}>0 \\
\frac{3}{4} z_{3}-\frac{1}{3} z_{4}>0 \\
-\frac{1}{4} z_{2}-\frac{1}{5} z_{3}+z_{4}>0
\end{array}
\end{array}\right\} .
$$

Let $z=(1,1,1,1)^{T} \in \Omega_{\varrho}\left(\mathcal{A}_{0}+\mathcal{A P}\right)$ and $\lambda=0.05$ which satisfies the inequality

$$
\left(\mathcal{A}_{0} e^{\lambda H_{1}}+\mathcal{A} \mathcal{P}^{\lambda H_{2}}-E\right) z \leq 0
$$

where, $1 \leq H_{1}=\int_{t-1}^{t}\left(1+\cos ^{2} t\right) d s \leq 2,2 \leq H_{2}=\int_{t-2}^{t}\left(1+\cos ^{2} t\right) d s \leq 4$.

Let $\gamma_{k}=3 \max \left\{\alpha_{1 k}+\beta_{1 k}, \alpha_{2 k}+\beta_{2 k}\right\}$, then $\gamma_{k}$ satisfy $\gamma_{k} z \geq R_{k} z, k=1,2, \ldots$

Case 4.1. Let $\alpha_{1 k}=\alpha_{2 k}=\frac{1}{9} e^{\frac{1}{25^{k}}}, \quad \beta_{1 k}=\beta_{2 k}=\frac{2}{9} e^{\frac{1}{25^{k}}}$ and $t_{k}-t_{k-1}=2 k$, then $\gamma_{k}=e^{\frac{1}{25^{k}}} \geq 1$ and

$$
\frac{\ln \gamma_{k}}{\int_{t_{k-1}}^{t_{k}} h(s) d s}=\frac{\ln \gamma_{k}}{\int_{t_{k-1}}^{t_{k}}\left(1+\cos ^{2} t\right) d s} \leq \frac{\ln e^{\frac{1}{25^{k}}}}{2 k}=\frac{1}{25^{k} \times 2 k} \leq 0.02=\gamma<\lambda .
$$

By simple computation, we know that $\sigma_{k}=e^{\frac{1}{25^{k}}} \geq 1$, $\mathcal{R}_{k}\left(E-\mathcal{A}_{0}-\mathcal{A P}\right)^{-1} \mathcal{A} \Lambda=\frac{1}{e^{25^{k}}}(0.3361,0.3810,0.3361,0.3810)^{T}, \quad \mathcal{R}_{k}\left(E-\mathcal{A}_{0}-\mathcal{A} \mathcal{P}\right)^{-1} \mathcal{A} \Lambda=e^{\frac{1}{25^{k}}}(0.3361,0.3810,0.3361,0.3810)^{T}$, $\sigma_{k}\left(E-\mathcal{A}_{0}-\mathcal{A P}\right)^{-1} \mathcal{A} \Lambda=e^{\frac{1}{25^{k}}}(1.2773,0.8739,1.2773,0.8739)^{T}$.

Clearly, all conditions of Theorem 3.2 are satisfied. So $S=\left\{\phi \in P C \mid-\left(1.2773 e^{\frac{1}{24}}, 0.8739 e^{\frac{1}{24}}\right)^{T} \leq \phi \leq\left(1.2773 e^{\frac{1}{24}}, 0.8739 e^{\frac{1}{24}}\right)^{T}\right.$ is a global attracting set of (42). 
Case 4.2. Let $\alpha_{1 k}=\alpha_{2 k}=\frac{1}{9} e^{\frac{1}{2^{k}}}$ and $\beta_{1 k}=\beta_{2 k}=0$, then $R_{k}=\frac{1}{9} e^{\frac{1}{2^{k}}} E \leq E$. Therefore, by Theorem 3.3, $S=\left\{\varphi \in P C \mid-(1.2773,0.8739)^{T} \leq \varphi \leq(1.2773,0.8739)^{T}\right.$ is a positive invariant set and also a global attracting set of (42).

Case 4.3. If $\mu=0$ and let $\alpha_{1 k}=\alpha_{2 k}=\frac{1}{3} e^{0.04 k}$ and $\beta_{1 k}=\beta_{2 k}=\frac{2}{3} e^{0.04 k}$, then $\gamma_{k}=e^{0.04 k} \geq 1$ and

$$
\frac{\ln \gamma_{k}}{\int_{t_{k-1}}^{t_{k}} h(s) d s}=\frac{\ln \gamma_{k}}{\int_{t_{k-1}}^{t_{k}}\left(1+\cos ^{2} t\right) d s} \leq \frac{\ln e^{0.04 k}}{2 k}=0.02=\gamma<\lambda .
$$

Clearly, all conditions of Corollary 3.1 are satisfied. Therefore, by Corollary 3.1, the zero solution of (42) is globally exponentially stable.

\section{Acknowledgements}

The authors would like to thank several anonymous reviewers for their constructive suggestions and helpful comments. The study was supported by the National Natural Science Foundation of China under Grants 10971147 and 11101367

\section{Author details}

${ }^{1}$ Department of Mathematics, Zhejiang International Studies University, Hangzhou, 310012, P. R. China ${ }^{2}$ Department of Applied Mathematics, Zhejiang University of Technology, Hangzhou, 310023, P. R. China

\section{Authors' contributions}

$\mathrm{DH}$ provided the main idea of this article and carried out the main proof of the theorems. QM carried out the proof of Theorem 3.1. All authors read and approve the final manuscript.

\section{Competing interests}

The authors declare that they have no competing interests.

Received: 4 February 2012 Accepted: 23 July 2012 Published: 23 July 2012

\section{References}

1. Deng, JQ: A note on oscillation of second-order nonlinear difference equation with continuous variable. J Math Anal Appl. 280, 188-194 (2003). doi:10.1016/S0022-247X(03)00068-4

2. Deng, JQ: Existence for continuous nonoscillatory solutions of second-order nonlinear difference equations with continuous variable. Math Comput Model. 46, 670-679 (2007). doi:10.1016/j.mcm.2006.11.028

3. Deng, JQ, Xu, ZJ: Bounded continuous nonoscillatory solutions of second-order nonlinear difference equations with continuous variable. J Math Anal Appl. 333, 1203-1215 (2007). doi:10.1016/j.jmaa.2006.12.038

4. Ladas, G: Recent developments in the oscillation of delay difference equations. Differential Equations. Lect Notes Prue Appl Math. 127, 321-332 (1991)

5. Philos, CHG, Purnaras, IK: An asymptotic result for some delay difference equations with continuous variable. Adv Diff Equ. 1, 1-10 (2004)

6. Philos, CHG, Purnaras, IK: On non-autonomous linear difference equations with continuous variable. J Diff Equ Appl. 12, 651-668 (2006). doi:10.1080/10236190600652360

7. Philos, CHG, Purnaras, IK: On the behavior of the solutions to autonomous linear difference equations with continuous variable. Arch Math (BRNO). 43, 133-155 (2007)

8. Peng, MS: Oscillation theorems of second-order nonlinear neutral delay difference equations with impulses. Comput Math Appl. 44, 741-748 (2002). doi:10.1016/S0898-1221(02)00187-6

9. Peng, MS: Oscillation criteria for second-order impulsive delay difference equations. Appl Math Comput. 146, 227-235 (2003). doi:10.1016/S0096-3003(02)00539-8

10. Zhang, QQ: On a linear delay difference equation with impulses. Ann Diff Equ. 18, 197-204 (2002)

11. Zhang, $\mathrm{H}$, Chen, LS: Oscillation criteria for a class of second-order impulsive delay difference equations. Adv Complex Syst. 9, 69-76 (2006). doi:10.1142/S0219525906000677

12. Zhang, Y, Sun, JT, Feng, G: Impulsive control of discrete systems with time delay. IEEE Trans Autom Control. 54 830-834 (2009)

13. Zhu, W, Xu, DY, Yang, ZC: Global exponential stability of impulsive delay difference equation. Appl Math Comput. 181, 65-72 (2006). doi:10.1016/j.amc.2006.01.015

14. Ma, ZX, Xu, LG: Asymptotic behavior of impulsive infinite delay difference equations with continuous variables. Adv Diff Equ. 1, 1-13 (2009)

15. Zhu, W: Invariant and attracting sets of impulsive delay difference equations with continuous variables. Comput Math Appl. 55, 2732-2739 (2008). doi:10.1016/j.camwa.2007.10.020

16. Chu, TG, Zhang, ZD, Wang, ZL: A decomposition approach to analysis of competitive-cooperative neural networks with delay. Phys Lett A. 312, 339-347 (2003). doi:10.1016/S0375-9601(03)00692-3 

journal and benefit from:

- Convenient online submission

$\checkmark$ Rigorous peer review

- Immediate publication on acceptance

- Open access: articles freely available online

- High visibility within the field

- Retaining the copyright to your article

Submit your next manuscript at $\boldsymbol{\nabla}$ springeropen.com 